\title{
La vetula di messer Niccolò
}

\section{Paolo Cherchi}

L'epistola è celebre: lo è per l'evento affatto singolare che Machiavelli racconta a Luigi Guicciardini e per il modo in cui viene esposto. Eccone il testo:

Affogaggine, Luigi; et guarda quanto la Fortuna in una medesima faccienda dà ad li uomini diversi fini. Voi, fottuto che voi havesti colei, vi è venuta voglia di rifotterla et ne volete un'altra presa; ma io, stato fui qua parecchi dí, accecando per carestia di matrimonio, trovai una vechia che m'imbucatava le camicie, che sta in una casa che è piú di mezo sotterra, né vi si vede lume se non per l'uscio. Et, passando io un dí di quivi, la mi riconobbe et, factomi una gran festa, mi disse che io fussi contento andare un poco in casa, che mi voleva mostrare certe camicie belle, se io le volevo comperare. Onde jo, nuovo cazo, me lo credetti, et, giunto là, vidi al barlume una donna con uno sciugatoio tra in sul capo et in sul viso, che faceva el vergognoso, et stava rimessa in un canto. Questa vechia ribalda mi prese per mano et, menatomi ad colei, dixe:-Questa è la camicia che io vi voglio vendere, ma voglio la proviate prima et poi la pagherete-. Io, come peritoso che io sono, mi sbigotti' tucto; pure, rimaso solo con colei et al buio (perché la vechia si uscí subito di casa et serrò l'uscio), per abreviare, la fotte' un colpo; et benché io le trovassi le coscie vize et la fica umida et che le putissi un poco el fiato, nondimeno, tanta era la disperata foia che havevo, che la n'andò. Et facto che io l'hebbi venedomi pure voglia di vedere questa mercatantia, tolsi un tizzone di fuoco d'un focolare che v'era et accesi una lucerna che vi era sopra; né prima el lume fu appreso, che 'l lume fu per cascarmi di mano. Omé! fu' per cadere per terra morto, tanta era bructa quella femina. E' se le vedeva prima un ciuffo di capelli fra bianchi et neri, cioè canuticci, et benché l'havessi el cocuzolo del capo calvo, per la cui calvitie ad lo scoperto si vedeva passeggiare qualche pidochio, nondimeno e pochi capelli et rari le aggiungnevano con le barbe loro infino in su le ciglia; et nel mezo de la testa piccola et grinzosa haveva una margine di fuoco, che la pareva bollata ad la colonna di Mercato; in ogni puncto delle ciglia di verso li ochi haveva un mazetto di peli pieni di lendini; li ochi haveva uno basso et uno alto, et uno era maggiore che l'altro, piene le lagrimatoie di cispa et nipitelli dipilicciati; il naso li era conficto sotto la testa arricciato in su, et l'una delle nari tagliata, piene di mocchi; la bocca somigliava quella di 
Lorenzo de' Medici, ma era torta da uno lato, et da quello n'usciva un poco di bava, ché, per non havere denti, non poteva ritenere la sciliva; nel labbro di sopra haveva la barba lunghetta, ma rara; el mento haveva lungo, aguzato et torto un poco in su, dal quale pendeva un poco di pelle che le aggiungneva infino ad la facella della gola. Stando adtonito ad mirare questo mostro, tucto smarrito, di che lei accortasi volle dire:-Che havete voi, messere?-; ma non lo disse, perché era scilinguata; et come prima aperse la bocca, n'uscí un fiato sí puzolente, che trovandosi offesi da questa peste due porte di dua sdegnosissimi sensi, li ochi e il naso, e' n'andò tale sdegno ad lo stomaco che, per non potere sopportare tale offesa, tucto si commosse, et commosso operò, sí che io le rece' addosso. Et cosí, pagata di quella moneta che la meritava, mi parti'. (1112)

Nessuno oggi, come del resto il destinatario di quel lontano 1509 , dà a questo testo un valore autobiografico ché tutto in esso ha sentore di esercizio letterario: la situazione è tipica da commedia e la descrizione della vecchia è troppo scopertamente condotto sul pattern di una vetusta tradizione. E proprio su quest'ultimo aspetto-la spia piú ovvia dell'origine letteraria dell'evento narrato-hanno insistito lettori attenti come il Basile (576-578), il Ferroni (215-264; in particolare 226-231), e il Martelli (226-231) che hanno rivisitato il topos della descriptio vetulae dalle artes dictandi mediolatine alle riprese umanistiche di un Poliziano. Mi sembra, però, che si possa compiere ancora un passo nella verifica dell'origine letteraria di questa "novelletta" indicando una fonte cui si potrebbe far risalire l'occasione che dà l'avvio a quello che si deve considerare come il vero nucleo della lettera machiavelliana, cioè la descrizione della laida vecchia.

Il testo cui alludo è il De vetula, un lungo poema che appartiene alla tradizione pseudo-ovidiana medievale, composto nel XIII secolo. Protagonista del poema è Ovidio che nel primo libro dell'opera vien presentato nel suo palazzo dedito agli amori facili e agli ozi e agli svaghi (quali l'equitazione e la caccia, la pesca e il nuoto) nonché al gioco d'azzardo o agli scacchi. Ma a mutare questa dolce vita interviene l'innamorato. Ovidio (e siamo al secondo libro dell'opera) s'invaghisce d'una giovane donna che, purtroppo, non gli si può concedere perché i genitori la sorvegliano. Ovidio ricorre allora ad una mezzana, una vecchia che era stata nutrice della giovane. Il poeta la persuade a prestarsi come ruffiana, e la vecchia non sa resistere al miraggio del compenso. Perciò ella prepara un convegno notturno in una stanza "extra maternos thalamos," dove l'amante potrà raggiun- 
gerla e possederla. La mezzana farì in modo di aprirgli la porti. Ovidio si prepara fin da buon mattino con cibi sostanziosi c con abbigliamento appropriato alla notte d'amore. Ed eceo, arrivata la notte, dopo un accidentato vagare per una casa labirintica sommersa nel buio, Ovidio, eccitatissimo, perviene alla stanza dell'amatá ed "explorante manu" cerca il letto:

En, humili strato quasi sompno pressa iacebat.

Quanta, putas, interna meas dulcedo medullas tunc demulceret! Quanto meus afficeretur tunc animus desiderio, non est leve dictu. Illico tollo moras, omnis dampnosa videtur quantumcumque brevis mora temporis, ocius ergo abicio vestes, sed et accelleratio tanta impedit accelerare meum, mora nascitur inde, unde moram vitare volens sic accelerabam. Abicio vestes subitoque preoccupo nudam circumplexus eam, nec ei divertere quoquam iam licet, omnis ei motus est forma negata; virginis artari sic vult pudor, et sibi parci, si non narcatur, reputat. Cum virgine nostra sic decuisset agi, si praesens ipsa fuisset, a cupido Iove sic Semelem decuisset adiri; ipsaque erat Beroe sic a Iove non adeunda.

(vv. 469-485)

Ma tanta gioia è offuscata da un imporvvisa rivclazione:

Heu michi, tanta meis regnans dulcedo medullis quam modicum mansit! Reperi contraria votis, vertitur in luctum cithare sonus inque stuporem deliciarum spes, moritur fax ignis amoris, Si quid erat, quod epar ventoso turbine misso fecerat arrectum, subito languetque caditque; sopitur virtus, frigescunt omnia membra. Credere quis posset, quod virgo quatuor implens nuper olympiades adeo cito consenuisset!

Numquam tam modico rosa marcuit. In novas formas corpora mutatas cecini, mirabiliorque non reperitur ibi mutatio quam fuit ista, scilicet, ut fuerit tam parvo tempore talis taliter in talem vetulam mutati puella. Heu quam dissimiles sunt virginis artubus artus! Accusant vetulam membrorum turba senilis, 
collum nervosum, scapularum cuspis acuta, saxossum pectus, laxatus pellibus uber, -non uber, sed tam vacuum quam molle, velut sunt burse pastorum,- -venter sulcatus aratro, arentes clunes macredine, crudaque crura inflatumque genu vincens adamanta rigore:

Accusant vetulam membrorum marcida turba.

(vv. 486-508)

Ovidio è tentato di ucciderla, ma si contiene per non esporre con lo scandalo l'amata. Rientra a casa e maledice la vecchia; e fra le altre maledizioni leggiamo queste:

Fletus ei sit continuus lacrimeque perennes, singultus subiti, suspiria crebra frequenter, oscitet alitibus distenta rigoribus atque feteat eructatio, non emungere nares possit, in os sanies descendat tota corize, nec spuat hoc etiam, sed glutiat evomitura.

(vv. 540-545)

Quest'episodio determina una svolta fondamentale nella vita di Ovidio che rinuncia all'amore e si dedica alla filosofia, tema che occupa il terzo libro del De vetula. Anche il titolo dell'opera, del resto, sottolinca l'importanza dell'episodio, c ogni lettore è guidato a coglierla. L'averlo seguito per esteso ci consente di vedere quanto fosse facile per un lettore quale Machiavelli ricavarne la novelletta (è il termine usato dal Martelli) della vecchia. Bastava immaginarsi come protagonista non innamorato come Ovidio ma altrettanto infoiato e isolare, pertanto, nell'originale il solo episodio dell'incontro al buio con la vecchia: nel caso di Machiavelli con una vecchia non conosciuta prima, mentre nel caso di Ovidio l'identità fra la mezzana c la vecchia, che si trova a letto è centrale alla fabula del poema. L'atteggiamento di autoderisione richiedeva quindi che l'incontro dell'originale venisse a concludersi con il ripetuto coito che lí mancava. E una volta introdotto questo nuovo elemento, la scoperta d'aver compagna di letto una vecchia non si dà nell'amplesso ma in una perlustrazione successiva fatta con la vista e l'odorato e non col tatto: sono quelli i sensi che permettono un piú ricco catalogo delle bruttezze della vecchia.

Rimane solo un problema. Machiavelli conosceva il De vetula? 
La notevole fortuna dell'opera che fu stampala per la prima volta a Perugia nel 1475 e di cui sopravvivono vari manoscritti in biblioteche italiane, e la curiosita del Machiavelli per la commedia elegiaca medioevale cui il De vetula è spesso associata nella tradizione manoscritta,' rendono plausibile una risposta positiva. Se non fosse cosí, dovremmo pensare che la combinatoria della mente umana è alquanto limitata, per cui cra possibile che l'autore del De l'etula c Machiavelli fossero, a distanza di sccoli, capaci di immaginare una situazione analoga che presenta la rara circostanza di trovarsi all insaputa con una vecchia rivoltante a letto in una stanza oscura, circostanza che provoca nel protagonista maschile disgusto fino al vomito una volta che si rende conto d'aver accarezzato o goduto una vecchia laida. ${ }^{2}$

The University of Chicago

\section{NOTE}

1 Per il census dei manuscritti e delle edizioni si rimanda all’ampio studio di P. Klopsch alla sua ed. citata.

2 Tanto il Basile quanto il Ferroni indicano una possibile fonte della novellella di Messer Niccolò in una novella del Decameron (VIII, 4). Ci sono, è verò delle similarità. Ma intanto Ciutazza, con cui giace il "prevosto," non c̀ una vecchia; e il disgusto per aver giaciuto con una donna brutta non fa parte del racconto boccaciano.

\section{OPERE CONSULTATE}

BASILE, B. "Grotteschi machiavelliani." Contivium 34 (1966): 576-83.

De vetula. Ed. Paul Klopsch. Leida-Colonia: Brill, 1967.

FERRONI, G. "Le 'cose vane' nelle lettere del Machiavelli," Rassegna della lelleratura italiana, 76 (1972): 215-264.

MARTELLI, M. Una giarda fiorentina. Roma: Salerno Editrice. 1978. 